\title{
Fabrication of functional structures on thin silicon nitride membranes
}

\author{
P. Ekkels ${ }^{\mathrm{a}, *}$, R.W. Tjerkstra ${ }^{\mathrm{a}}$, G.J.M Krijnen ${ }^{\mathrm{a}}$, J.W. Berenschot ${ }^{\mathrm{a}}$, J. Brugger ${ }^{\mathrm{b}}$, \\ M.C. Elwenspoek ${ }^{\mathrm{a}}$ \\ ${ }^{\mathrm{a}}$ Transduction Technology Group, MESA + Research Institute, University of Twente, P.O. Box 217, 7500 AE Enschede, \\ The Netherlands \\ ${ }^{\mathrm{b}}$ EPFL-STI-IMM; BM 3-116 1015 Lausanne, Switzerland
}

\begin{abstract}
A process to fabricate functional polysilicon structures above large $\left(4 \times 4 \mathrm{~mm}^{2}\right)$ thin $(200 \mathrm{~nm})$, very flat LPCVD silicon rich nitride membranes was developed. Key features of this fabrication process are the use of low-stress LPCVD silicon nitride, sacrificial layer etching, and minimization of membrane stress and deformation after fabrication, which was done by complete removal of the sacrificial layer, avoidance of sharp corners in the design of the functional polysilicon structures, and annealing of the polysilicon after doping. A polysilicon layer that was directly attached to the silicon nitride membrane was used. The polysilicon was doped locally to separate the rotors from the stators electrically, preventing short-circuiting. The layer was patterned with a hexagonal supporting structure that serves three main functions: membrane strengthening, electrical wiring and microactuation. As a demonstration of the technology a pseudo hexagonal polysilicon framework with embedded comb-drive actuated microshutter suspended above a $\sim 150$-nm thick membrane, was successfully fabricated.
\end{abstract}

(C) 2003 Elsevier Science B.V. All rights reserved.

Keywords: Nanopatterning; Surface micromachining; Electrostatic actuation

\section{Introduction}

In the quest for faster and more powerful computers with increased memory, the use of submicrometer structures in integrated electronic circuits is already common. These structures are often made using photolithographic techniques. An important drawback of the use of photolithography, associated with the (repeated) cycle of photoresist deposition, illumination and removal,

\footnotetext{
*Corresponding author. Tel.: +31-53-489-4438/2751; fax: +31-53-489-2831.
}

E-mail address: p.ekkels@el.utwente.nl (P. Ekkels). 
is its sensitivity to contamination making it less favourable for fabrication of very delicate structures. Hence, new techniques suitable to make nanometer-sized structures on silicon or other materials are of much interest. Moreover, there is a need for high-resolution deposition and patterning techniques of unconventional materials such as biomolecules for applications as biochips.

Local deposition, preferably under very clean conditions in ultra high vacuum systems, is promising for fabrication of structures with high resolution and of structures of high purity. A technique providing these possibilities is nanostencilling [1]. In this technique thin perforated membranes are positioned between an evaporation source and a sample in a UHV environment. The perforated membrane acts as a shadow mask and if the thickness of the membrane and the separation with the sample are small and the distance to the evaporation source is large, the pattern yielded by local deposition will resemble the perforation of the membrane to a large extent. One of the main advantages of evaporating through a shadow mask is the fact that the surface of the sample stays clean and that no material needs to be removed after deposition. Therefore, vulnerable samples that would not survive lithographic processes can also be patterned. During evaporation through a nanostencil, patterns can be made by moving the sample [2]. By changing the speed of the sample, the thickness of the deposited wires can be varied locally. Still more possibilities will arise when using multiple evaporation sources.

With the addition of microshutters, selectively and locally covering openings in the nanostencil, many new possibilities can be created depending on the shape of the shutters, the underlying perforation pattern and the dynamic operation during deposition. The fabrication of single electron transistors and other quantum devices will be greatly facilitated when microactuated shutters are combined with perforated membranes to obtain active nanostencils. Within the European project assembler tool for molecular structures (IST-1999-14192, ATOMS) we have set out to fabricate such active nanostencils. Building on technology developed for the fabrication of micro- and nanosieves [3], successful evaporation of gold dots through static nanostencils has been demonstrated before [1]. The extension of this research to active nanostencils is the topic of this paper. The design of a fabrication process and its successful application to fabrication of microactuators integrated with nanostencils is described.

\section{Process development}

\subsection{Active stencil architecture}

Starting point for the process development is the basic idea of combining silicon based bulk micromachining of membranes [3] with surface micromachining of polysilicon active structures [4]. Since the latter can hardly be carried out on deeply recessed structures and since the sample-tomembrane distance needs to be extremely small $(<100 \mathrm{~nm})$ for nanostencilling the only viable architecture seems to be the one in which the sample approaches the active stencil from the backside of the chip (see Fig. 1). However, this calls for rather large membranes in order to allow for reasonably sized, and thus easy to manipulate samples. Within ATOMS membrane sizes of $4 \times 4 \mathrm{~mm}^{2}$ is set as standard. Membranes of these sizes being only 100-200 nm thick are very vulnerable for process and handling induced stress. Therefore a polysilicon layer is deposited directly onto the thin $\mathrm{SiN}$ membrane. The first function of the polysilicon layer is to increase the membrane strength by 


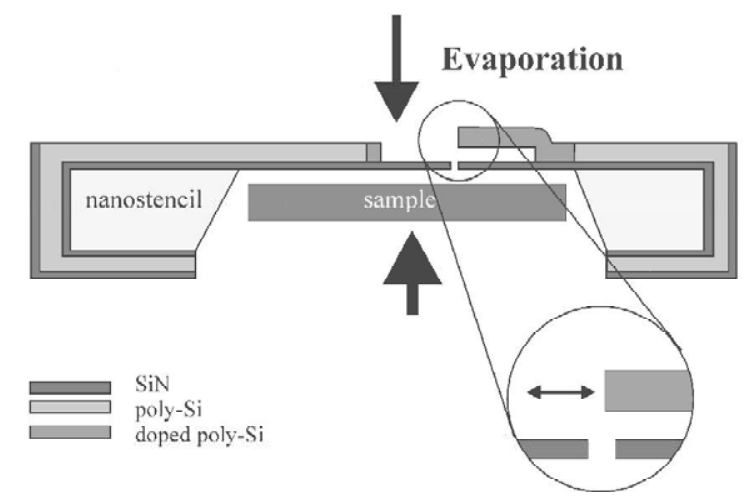

Fig. 1. Schematic representation of the use of microshutters in nanostencilling.

supplying a support. A second function of the polysilicon layer is to provide electrical connections to individual structures. Since membrane strengthening and electrical wiring are conflicting when the polysilicon layer is overall conductive, the need for local doping of the polysilicon layer (which is undoped during deposition) was recognized. The microshutters or other functional elements are formed in this polysilicon layer. Considering the difficulties of stress and temperature control in UHV thermal actuation [4] was abandoned, as was electromagnetic actuation for reason of the difficulties to produce high-count turn inductors. Due to its relative simplicity over piezoelectric actuation and its favorable scaling properties [5] electrostatic actuation was eventually chosen.

\subsection{Stress control}

For nanostencilling the sample to membrane distance should be very small to obtain small features on the sample from small perforations in the membrane. This also implies that stress in the layer package should be minimised in order to prevent bending, or even buckling which would lead to (locally) increased separation between membrane and sample. Preliminary experiments with a polysilicon- $\mathrm{SiO}_{2}$-low-stress $\mathrm{SiN}$ [6] layer package revealed large deformations yielding unusable membranes. To investigate this stress, experiments were carried out with various layer packages and fabrication conditions. These experiments indicated that the $\mathrm{SiO}_{2}$ (TEOS) layer, exposed to the high temperature steps required for the diffusion of boron in the polysilicon layer, was the main source of stress [7]. In order to avoid the stress resulting from the $\mathrm{SiO}_{2}$ layer an approach was used in which $\mathrm{SiO}_{2}$ is completely eliminated in the final structure, reducing the layer package to two-layers with the polysilicon directly anchored on the $\mathrm{SiN}$.

\subsection{Electrical connection and local doping}

A simple solution of the problem of electrical connections of the shutters on the membrane is the physical separation of the individual electrical conductors. In this case, the polysilicon layer is doped uniformly and by etching away the polysilicon between two conductors they become electrically isolated. However, this approach was abandoned because of the detrimental effects it would have on 
the overall strength of the polysilicon pattern. An alternative approach is local doping of polysilicon. During boron diffusion $\mathrm{S} \mathrm{SiO}_{2}$ mask is used to prevent diffusion of boron into the polysilicon locally. Electrical isolation between the individual contacts is obtained by the relatively low conduction of nondoped polysilicon. To determine the required dimensions for isolation a polysilicon line of $4-\mu \mathrm{m}$ thickness was investigated for various diffusion barrier widths. The results presented in Fig. 2 show that a barrier width of $30 \mu \mathrm{m}$ is sufficient to isolate two individual electrical areas after annealing. Using white light interference microscopy (WLIM) it was experimentally verified that local doping gave only rise to small $(20-50 \mathrm{~nm})$ deformations of the membrane which can be further minimised by proper design of the polysilicon structures.

\subsection{Process outline}

The fabrication process for the active membranes is as follows (Fig. 3). In order to get flat $\mathrm{SiN}$ membranes a 200-nm SiN layer is deposited directly onto a $\langle 100\rangle$ silicon wafer (A). Next, the SiN layer is covered with $\mathrm{SiO}_{2}$ layer of $1.5 \mu \mathrm{m}$ by TEOS deposition and subsequently patterned (B). After patterning, the $\mathrm{SiO}_{2}$ is only present at positions where free-standing parts of the actuators are required. Then a polysilicon layer of $4.5 \mu \mathrm{m}$ is deposited (C), doped locally with boron by solid source boron dotation (D) (using a PECVD oxide mask not shown in Fig. 3), and patterned by RIE-etching (E). In the next steps the membrane is formed using $\mathrm{KOH}$ etching $(\mathrm{F})$. This approach is preferred to methods that use dry etching which show a poor etch-stop on the $\mathrm{SiN}$ membrane or one-sided wet-etching which is vulnerable for leakage. In order to protect the polysilicon during $\mathrm{KOH}$ etching the structures are covered with a $100-\mathrm{nm}$ SiN protection layer which is removed later by RIE-etching after the membranes are etched open from the backside. Finally, the actuators are etched free in a BHF solution $(\mathrm{G})$ during sacrificial layer etching. After this step the actuators are rinsed thoroughly with water and IPA, and subsequently immersed in cyclohexane. Then, they are released using freeze-drying [8].

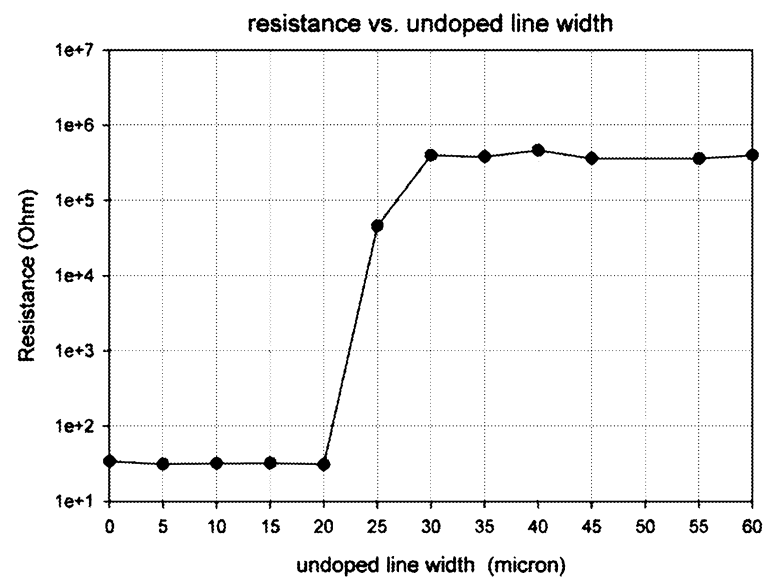

Fig. 2. Resistance versus undoped line width for a 4- $\mu \mathrm{m}$ thick polysilicon layer. 
A.

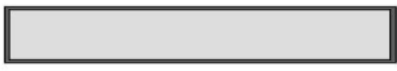

B.

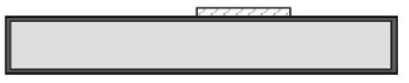

C.

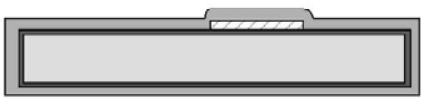

D.

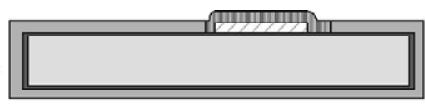

E.

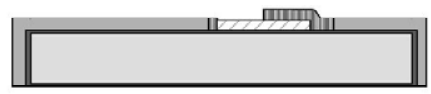

F.

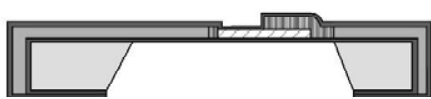

G.
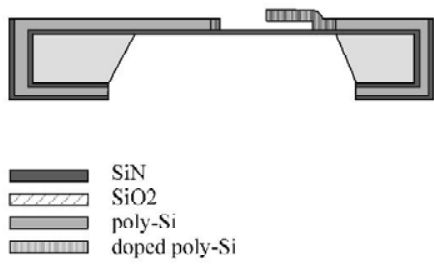

Fig. 3. Abbreviated process steps for fabrication of nanostencil.

\section{Design}

\subsection{Hexagonal basis structure}

The polysilicon layer is subdivided in a pattern of hexagonal basis cells. Each individual cell can be given a specific function. In this work we only present cells filled with microactuators but other functionality can be obtained, e.g. by including mechanical proximity sensors in certain cells. The hexagonal cells consist of beams of 50 $\mu \mathrm{m}$ width and 115-170 $\mu \mathrm{m}$ length and provide three functions:

1. The polysilicon framework prevents the membrane from deforming and/or breaking under heavy load. It prevents the propagation of cracks in the silicon nitride. It also helps to balance the high stress levels that arise in the sacrificial oxide layer during processing.

2. The polysilicon provides electrical connection to the individual cells. Since no crossing connections can be made in our technology the cells are connected in series to form columns of actuators operating on one and the same potential difference. To prevent short-circuiting $30 \mu \mathrm{m}$ wide diffusion barriers were used during local doping. The size of the barriers is relatively small, avoiding deformation of the membrane.

3. Within one cell a microactuator is anchored on the hexagonal framework only (i.e. not on the membrane). This way deformation of the membrane underneath the actuators is prevented.

A schematic representation of the design of the pattern of hexagonal cells of polysilicon is shown in Fig. 4.

\subsection{Microactuators}

To demonstrate the applicability and functionality of our approach a design of a simple electrostatic comb-drive structure, fitting into a basis-cell, has been implemented. The comb drive was designed for 


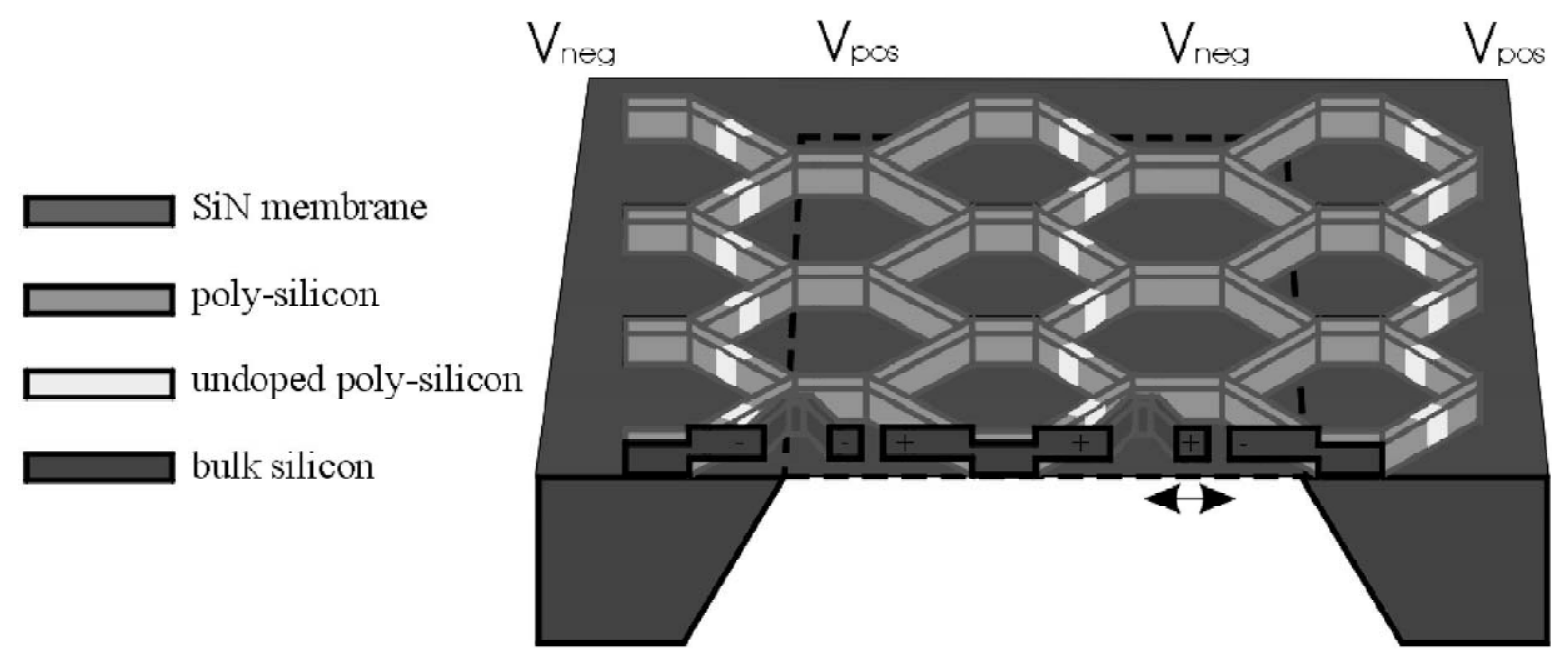

Fig. 4. Schematic representation of the voltage distribution over the hexagonal cells of the polysilicon supporting structure.

small $(<2 \mu \mathrm{m})$ movements at low voltage. It consists of a single comb actuator suspended by a folded-spring structure. The structure is anchored to the hexagonal framework on two points which are electrically isolated from each other. The dimensions of the actuator/spring design are the result of an optimisation of surface area use of the actuators. The vertical stiffness of the structure is high thus minimising the chance of stiction between actuator and membrane.

The displacement of the comb-fingers as a function of the comb-drive parameters and the spring-stiffness $k_{\mathrm{s}}$ is given by:

$$
x=\frac{n_{\mathrm{c}} \varepsilon_{0} h}{d k_{\mathrm{s}}} \cdot V^{2}
$$

with $n_{\mathrm{c}}$ the number of fingers, in this case 29, $d$ the distance between the fingers of the comb, $h$ the height of the comb-fingers, $V$ the voltage between the fingers and $e_{0}$ the permittivity of vacuum. To make optimal use of the area in a cell a double spring is folded 14 times to get a stiffness $k_{\mathrm{s}}$ of:

$$
k_{\mathrm{s}}=\frac{2}{14} \cdot \frac{3}{L^{3}} \cdot E_{\mathrm{Si}} \cdot \frac{h w^{3}}{12}
$$

with $L$ the length of a single spring, $h$ the thickness of the spring, $w$ the width of the spring and $E_{\mathrm{Si}}$ the Young's modulus of polysilicon. Using $2-\mu \mathrm{m}$ wide polysilicon beams and $2-\mu \mathrm{m}$ wide gaps the microactuator is designed to have a displacement of $1.6 \mu \mathrm{m}$ at a voltage of $20 \mathrm{~V}$.

\section{Results and discussion}

After the membranes are etched open (Fig. 3F) they are highly deformed. Due to the compressive stress of the $\mathrm{SiO}_{2}$ sacrificial layer, deformations of up to $100 \mu \mathrm{m}$ have been measured. After the sacrificial etch of $\mathrm{SiO}_{2}$ the membranes return to an almost perfectly flat state. The scanning electron microscope (SEM) images in Fig. 5 show a large part of the membrane (left) and a close up of one of 

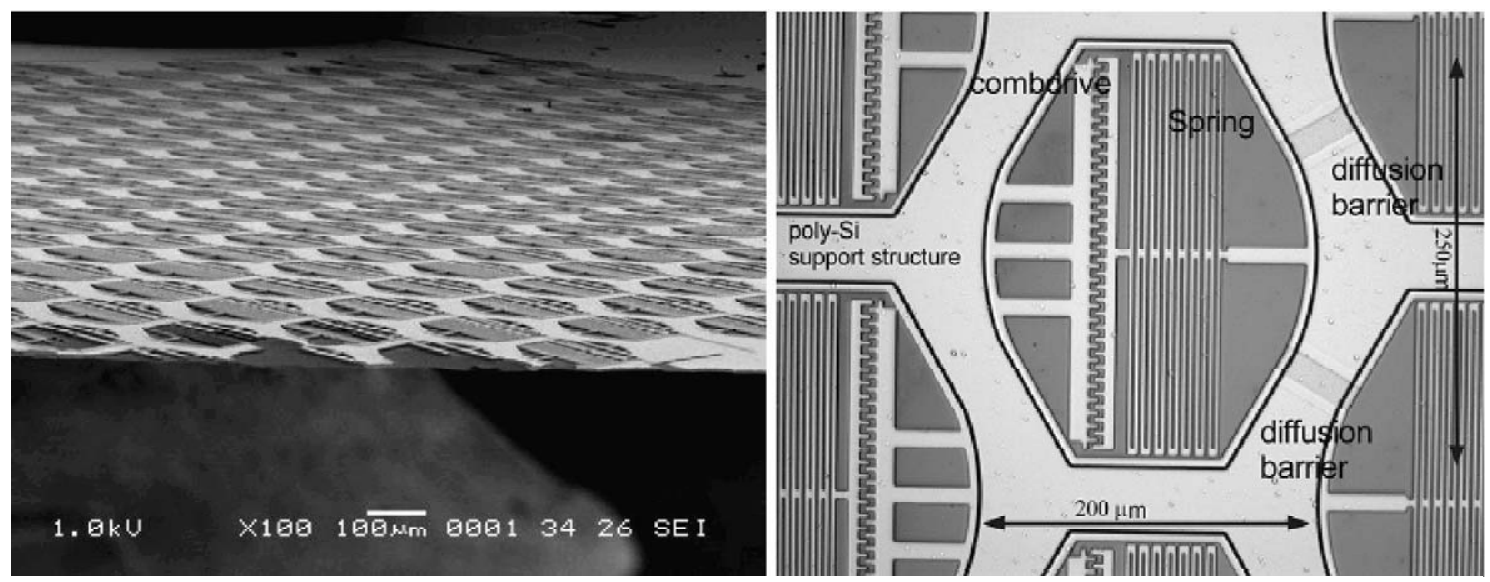

Fig. 5. Left: SEM picture of side view of membrane. SEM picture of single cell with actuator.

the microactuator cells (right), both indicating successful fabrication with very low (local) deflection. The curvature of the membranes was measured using white light interferometry (Fig. 6). Overall curvature over a quarter of the membrane was $50 \mathrm{~nm}$ with steps of $100 \mathrm{~nm}$ of the SiN between the hexagonal patchwork (Fig. 6, right). However, it is unclear whether the measurements only reveal the real membrane deflection or that artefacts, caused by multiple reflections on the air-SiN-air and the air-SiN-polysilicon layer packages, influence the measurements. In any case, in measurements using SEM no deformations could be detected. The hexagonal structure turns out to be a good way to prevent damaging of entire membranes. Imperfections in the framework were seen to be limited at most to neighbouring cells (see Fig. 6, left). Individual actuator arrays were actuated over a distance of about $1 \mu \mathrm{m}$ with $20 \mathrm{~V}$ peak to peak AC signal. The observed resonance frequency was in the order of $20 \mathrm{kHz}$. Operation with DC voltages resulted in pull-down of the actuators to the membrane. We believe this is caused by electrostatic charging of the SiN layer [9].
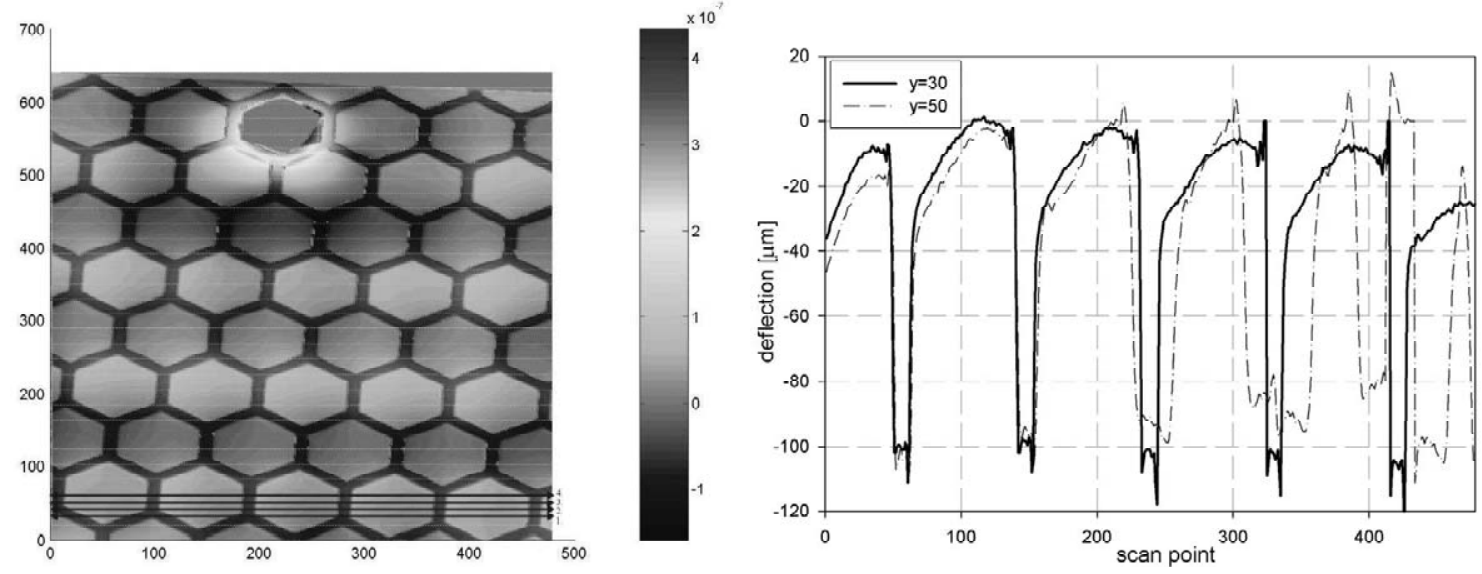

Fig. 6. Deflection measurements by white light interferometer microscopy of a quarter of the membrane showing the impact of a single damage on the membrane. 


\section{Conclusions and further developments}

In this paper a fabrication process to realize functional structures above thin $\mathrm{SiN}$ membranes has been proposed and demonstrated. Free standing polysilicon structures were fabricated by sacrificial layer etching on top of a $\mathrm{SiN}$ membrane. Stress in the final structures was minimised by complete removal of the sacrificial $\mathrm{SiO}_{2}$ and by anchoring the polysilicon structures directly to the $\mathrm{SiN}$ membranes. After local doping we were able to use the polysilicon layers for mechanical strengthening, electrical conduction and micro-actuation at the same time. As an example of the potential of the technology patterns of hexagonal structures with embedded electrostatic comb-drive structures were designed, fabricated and tested. Future work will comprise the incorporation of holes in the membranes by focused ion beam and the demonstration of the functionality of this enhanced nanostencil technology using deposition experiments.

Extended functionality of the membrane can be obtained by designing cells with different functionalities, e.g. for proximity detection (using piezoresistive elements), perforations on microscale (e.g. for registration markings on sample). Furthermore the proposed technology in principle allows for fabrication of integrated $2 \mathrm{D}$ scanning microactuators. This could be a very attractive approach compared to scanning the sample relative to the membrane because of the extremely small membrane to sample distances required for nanostencilling.

\section{Acknowledgements}

We would like to thank Meint de Boer for his assistance with polysilicon etching, and Matthijn de Rooij for his support with white light interferometer measurements. This work was supported by the European Union in the fifth framework programme project ATOMS (IST-1999-14912).

\section{References}

[1] J. Brugger, J.W. Berenschot, S. Kuiper, W. Nijdam, B. Otter, M. Elwenspoek, Microelectron. Eng. 53 (2000) $403-405$.

[2] R. Lüthi, R.R. Schlittler, J. Brugger, P. Vettiger, M.E. Welland, J.K. Gimzewski, Appl. Phys. Lett. 75 (1999) 1314-1316.

[3] J.M. van Rijn, G.J. Veldhuis, S. Kuiper, Nanotechnology 9 (1998) 343-345.

[4] N. Belov, V. Vaganov, S. in 't Hout, Proc. Eurosensors XIII, The Hague, The Netherlands, 13P4, 1999, pp. 489-492.

[5] R. Legtenberg, A.W. Groeneveld, M.C. Elwenspoek, J. Micromech. Microeng. 6 (1996) 320-329.

[6] J.G.E. Gardeniers, H.A.C. Tilmans, C.G.C.J. Visser, Vac. Sci. Technol. A 14 (1996) 2879-2892.

[7] S. Deladi, M.J. de Boer, D. Rosén, G. Krijnen, M.C. Elwenspoek, MicroMechanics Europe, (2002), Sinaia, Romania, in preparation.

[8] R. Legtenberg, H.A.C. Tilmans, J. Elders, M. Elwenspoek, Sens. Actuat. A 43 (1-3) (1994) $230-238$.

[9] J. Wibbeler, G. Pfeifer, M. Heitschold, Sens. Actuat. A 71 (1998) 74-80. 